\title{
Developing Marketing Model Canvas as an Assessment Tool for Marketing Curriculum
}

\author{
Kamran Ahmed Siddiqui \\ College of Business Administration, Imam Abdulrahman Bin Faisal University, Saudi Arabia
}

Received March 11, 2021; Revised April 17, 2021; Accepted May 23, 2021

\section{Cite This Paper in the following Citation Styles}

(a): [1] Kamran Ahmed Siddiqui, "Developing Marketing Model Canvas as an Assessment Tool for Marketing Curriculum," Universal Journal of Educational Research, Vol. 9, No. 6, pp. 1333-1339, 2021. DOI: 10.13189/ujer.2021.090623.

(b): Kamran Ahmed Siddiqui (2021). Developing Marketing Model Canvas as an Assessment Tool for Marketing Curriculum. Universal Journal of Educational Research, 9(6), 1333-1339. DOI: 10.13189/ujer.2021.090623.

Copyright $\mathrm{C} 2021$ by authors, all rights reserved. Authors agree that this article remains permanently open access under the terms of the Creative Commons Attribution License 4.0 International License

\begin{abstract}
This paper aims to share the experiences of designing a new all-inclusive assessment tool for marketing curriculum. For decades marketing plan has been considered as a universally acceptable component of marketing curriculum. Normally it has the highest weightage in the list of assessment items for any marketing syllabus at undergraduate, graduate, and executive education. But assessing marketing plan itself is considered as a difficult job by academics. This paper proposes all-inclusive, one-page Marketing Model Canvas as a tool for assessing marketing plan curriculum. It has seven parts; Customers; Competitors; Value Proposition; Product and Branding; Place and Distribution; Pricing; and Promotion.
\end{abstract}

Keywords Marketing Model Canvas, Marketing, Curriculum, Business Model Canvas

\section{Introduction}

Throughout the world 'Marketing Plan' is considered as an integral part of marketing curriculum and most common element at different levels marketing curriculum i.e., undergraduate, graduate, and doctoral programs. It has also been considered as an essential component of experiential learning methodology employed by business education.

Teaching an experiential learning-based course is considered as a hall-mark of top business school faculty. It takes time to learn how to get the most out of these marketing plans not only for the students but also for the faculty.

Assessing marketing plans in the classroom environment was always considered as a problem especially at the undergraduate levels [1]. Business School faculty was always in search of an assessment tool to evaluate the students' work. One of the approaches used to evaluate the marketing plan was the assessment through rubrics but detailed rubrics for assessing marketing plan was never thought and practiced. This paper provides a new robust and all-inclusive tool to assess marketing plans in the classroom environment.

Wharton Business School launched the first Marketing course titled as "Marketing Products" in 1904 and launched additional courses on advertising and salesmanship in 1909.[2] Other institutions like Harvard Business School, University of Wisconsin, Ohio State University, Northwestern University also made significant contributions toward the first marketing courses. [3]

Harvard Business School has been known for its initiative of incorporating experiential learning (Case method teaching) methodology in business education [4]. Similarly, MIT Sloan School of Management-have practiced "action learning" for decades [5]. In search of excellence their faculty members realized that there were no textbooks suitable to a graduate program in business. Their first solution to this problem was to interview leading practitioners of business and to write detailed accounts of what these managers were doing [6]. Now experiential learning is part of approximately $90 \%$ of full-time business education. This curriculum change is being universally accepted across MBA programs, and experiential learning 
is an integral part of all full-time MBA programs [7].

In business curriculum especially in marketing courses experiential learning (including case method teaching and marketing plan as assignment) has been driven by market forces, and not by internal business school initiatives to improve student learning. [8].

Marketing plan is a written document that summarizes what the marketer has learned about the marketplace, indicates how the firm plans to reach its marketing objectives, and helps direct and coordinate the marketing effort [9]. As an academic assignment 'Marketing plan' can be classified into three classes [10]:

- Develop marketing plan for a live-client,

- Develop marketing plan using a written case setting, or

- Develop marketing plan for a real product using publicly available information or data.

Regardless of the type of marketing plan, it is considered as the most important component of course assessment. In some cases, market plan as a single assignment captures a weightage $40 \%$ of the total course assessment. Table 1 shows various examples of marketing plan and its weightage in marketing curriculum assessment.

\section{Materials and Methods}

The objective of the current study was to develop a robust validated customized scale to be used in marketing curriculum. The entire procedure was based on the notion of "adoption" to "adaptation" originated by the seminal work of Alexander Osterwalder's Business Model Canvas [22] [23] [24]. Accordingly proposed Marketing Model Canvas has been divided into ten sections; Marketing Objective section explains the purpose of the marketing plan; Customers section including customer needs and wants, customer segment and customer profile; Competitors section including major competitors, strategies for competition including leader's, challenger's and follower's strategies and competitive tools; Value Proposition section including unique selling proposition, positioning objective and perceptual maps; Product section including product line objectives, product mix description, Branding section illustrates brand development objectives, brand sponsorship objectives, branding elements brand equity measures and brand portfolio role; Place section including distribution objectives and distribution network; Pricing section including pricing objectives, competitor's prices, retail price, promotional pricing and discounts; and Promotion section includes advertising objective, advertising media selection, digital media tools, sales promotion tools, PR tools, Sales Force utilization and event sponsorship. Financial Implications section explains where the cost streams (what is incurred to operate the marketing model) and the revenue stream (the cash gained from each customer segments) should be considered. These sections were identified with the help of earlier studies [25] [26] [27] [28].

Table 1. Summary of Courses with Marketing Plan as a \% of course assessment

\begin{tabular}{|c|c|c|c|}
\hline Institution \& Country & Course Title & Program & $\begin{array}{c}\text { Marketing Plan } \\
\text { as \% of course } \\
\text { assessment }\end{array}$ \\
\hline Harvard Business School, USA & Marketing Management & Graduate & $25 \%[11]$ \\
\hline DeGroote School of Business, Canada & Applied Marketing Management & Undergraduate & $25 \%[12]$ \\
\hline Rutgers School of Business USA & Strategic Marketing Planning & Graduate & $25 \%[13]$ \\
\hline University of South Australia, Australia & Marketing Practicum & Graduate & $30 \%[14]$ \\
\hline IESE Business School, Spain & Marketing Planning \& Implementation & Executive & $30 \%[15]$ \\
\hline University of Wellington, New Zealand & International Marketing & Graduate & $30 \%[16]$ \\
\hline Western University, Canada & Fundamentals of Marketing & Undergraduate & $20 \%[17]$ \\
\hline University of Wellington, New Zealand & Strategic Marketing & Executive & $20 \%[18]$ \\
\hline Sheridan College, Canada & Marketing Analysis and Planning & Undergraduate & $40 \%[19]$ \\
\hline University of Canterbury, Canada & Retail Marketing & Undergraduate & $40 \%[20]$ \\
\hline Georgian College, Canada & Marketing on the Web & Undergraduate & $40 \%[21]$ \\
\hline
\end{tabular}




\subsection{Validity}

In the second phase validity was ensured through a panel of eight faculty members from different business schools, teaching marketing subjects at different levels i.e., undergraduate, MBA and executive education. Table 2 shows tabular presentation of respondents' demographic data. Typical validation sessions were started with a presentation stating the objective of the research, problems in assessing marketing plans and a need to make one-page, all-inclusive Marketing Model Canvas similar on the business model canvas. They were presented the initial draft of the model and solicited their suggestions.

A few suggestions were incorporated in the subsequent drafts of the model i.e., inclusion of customer needs and wants in customer section, perceptual maps in value proposition section and addition of digital media in promotion section.

After two weeks a follow-up meeting was held and different suggestions were incorporated in the final draft and presented to the respondents. Table 3 shows individual validity of each section obtained through validity tests.

\subsection{Pilot Testing}

At the final stage Marketing Model Canvas was tested at a leading business school. It was included in three marketing courses during the fall and spring semesters of academic year 2020-2021. Students have been adequately taught to prepare a marketing plan as part of their curriculum. They were given semester long duration to complete this assignment and present in front of their class fellows. Table 4 presents the results of pilot test for the Marketing Model Canvas.

Table 2. Respondent's demographic data

\begin{tabular}{|c|c|c|c|c|c|c|c|c|}
\hline \multirow{2}{*}{ Demographics } & \multicolumn{9}{|c|}{ Respondent's Data } \\
\cline { 2 - 18 } & $\mathbf{1}$ & $\mathbf{2}$ & $\mathbf{3}$ & $\mathbf{4}$ & $\mathbf{5}$ & $\mathbf{6}$ & $\mathbf{7}$ & $\mathbf{8}$ \\
\hline Age (Groups) & $25-35$ & $25-35$ & $35-45$ & $35-45$ & $35-45$ & $35-45$ & $45-55$ & $45-55$ \\
\hline Gender (M-Male; F-Female) & $\mathrm{F}$ & $\mathrm{F}$ & $\mathrm{M}$ & $\mathrm{M}$ & $\mathrm{M}$ & $\mathrm{M}$ & $\mathrm{M}$ & $\mathrm{M}$ \\
\hline Education (Levels) & Master & Master & $\mathrm{PhD}$ & $\mathrm{PhD}$ & $\mathrm{PhD}$ & $\mathrm{PhD}$ & $\mathrm{PhD}$ & $\mathrm{PhD}$ \\
\hline Experience (Years) & $1-5$ & $1-5$ & $5-10$ & $5-10$ & $10-15$ & $10-15$ & $15-20$ & $>25$ \\
\hline
\end{tabular}

Table 3. Respondent's Validity Check

\begin{tabular}{|c|c|c|c|c|c|c|c|c|}
\hline \multirow{2}{*}{ Section } & \multicolumn{8}{|c|}{ Respondent's Validity Check } \\
\hline & 1 & 2 & 3 & 4 & 5 & 6 & 7 & 8 \\
\hline Customers & $\sqrt{ }$ & $\sqrt{ }$ & $\sqrt{ }$ & $\sqrt{ }$ & & $\sqrt{ }$ & $\sqrt{ }$ & \\
\hline Competitors & $\sqrt{ }$ & $\sqrt{ }$ & $\sqrt{ }$ & $\sqrt{ }$ & $\sqrt{ }$ & & $\sqrt{ }$ & \\
\hline Value proposition & $\sqrt{ }$ & $\sqrt{ }$ & & $\sqrt{ }$ & $\sqrt{ }$ & $\sqrt{ }$ & & \\
\hline Product \& Branding & $\sqrt{ }$ & $\sqrt{ }$ & $\sqrt{ }$ & $\sqrt{ }$ & $\sqrt{ }$ & $\sqrt{ }$ & $\sqrt{ }$ & $\sqrt{ }$ \\
\hline Place & $\sqrt{ }$ & $\sqrt{ }$ & $\sqrt{ }$ & $\sqrt{ }$ & $\sqrt{ }$ & $\sqrt{ }$ & $\sqrt{ }$ & $\sqrt{ }$ \\
\hline Pricing & $\sqrt{ }$ & $\sqrt{ }$ & $\sqrt{ }$ & $\sqrt{ }$ & $\sqrt{ }$ & $\sqrt{ }$ & $\sqrt{ }$ & $\sqrt{ }$ \\
\hline Promotion & $\sqrt{ }$ & $\sqrt{ }$ & $\sqrt{ }$ & $\sqrt{ }$ & $\sqrt{ }$ & $\sqrt{ }$ & $\sqrt{ }$ & $\sqrt{ }$ \\
\hline
\end{tabular}

Table 4. Marketing Model Canvas - Pilot Test Results

\begin{tabular}{|c|c|c|c|c|}
\hline Course Title & Level & $\begin{array}{c}\text { No of } \\
\text { Participants }\end{array}$ & $\begin{array}{c}\text { Major } \\
\text { problems }\end{array}$ & $\begin{array}{c}\text { Submission } \\
\text { Rate }\end{array}$ \\
\hline Principles of Marketing & Undergraduate & 40 & Nil & $100 \%$ \\
\hline Marketing Management & MBA & 31 & Nil & $100 \%$ \\
\hline Strategic Marketing & EMBA & 7 & Nil & $100 \%$ \\
\hline
\end{tabular}




\begin{tabular}{|c|c|c|c|c|c|c|}
\hline \multicolumn{3}{|l|}{ Objective: } & \multicolumn{4}{|l|}{ Brand: } \\
\hline CUSTOMER NEEDS & COMPETITORS & VALUE PROPOSITIONS & PRODUCT STRATEGIES & PLACE STRATEGIES & \multicolumn{2}{|c|}{ | PROMOTION STRATEGIES } \\
\hline & Market Leader & Positioning Objective & Product Line Objective & Distribution Objective & \multicolumn{2}{|c|}{ Advertising Objective } \\
\hline & Market Challenger & $\square$ Positioning & $\square$ Line Stretching & IIntensive & \multicolumn{2}{|l|}{$\square$ Informative } \\
\hline & Market Follower & Q Re-Positioning & Q Line Filling & $\square$ Selective & \multicolumn{2}{|l|}{$\square$ Persuasive } \\
\hline & MAJOR COMPETITORS & Q De-Positioning & D Line Modemization & $\square$ Exclusive & \multicolumn{2}{|l|}{$\square$ Reminder } \\
\hline & Competitor $\# 1$ & Unique Selling Proposition & $\square$ Line Featuring & Distribution Network & \multicolumn{2}{|l|}{$\square$ Reinforcement } \\
\hline & Competitor $\# 2$ & USP $\# 1$ & $\square$ Line Pruning & $\square$ Superstores & \multicolumn{2}{|l|}{ Advertising Media } \\
\hline \multirow{7}{*}{ CUSTOMER WANTS } & Competitor $\# 3$ & $\mathrm{USP}=2$ & Product Mix & $\square$ Supermarket & D Broadcast - TV & \\
\hline & Competitor $\# 4$ & USP $\# 3$ & Product Mix - Width & $\square$ Convenience Store & a Broadcast Radio & \\
\hline & \begin{tabular}{|l|} 
COMPETITIVE STRATECIES \\
\end{tabular} & Perceptual Maps & Product Line - Length & $\square$ Discount Store & $\square$ Print-Newspaper & \\
\hline & Leader Strategies & & Product Item - Depth & $\square$ Department Store & $\square$ Print-Magazine & \\
\hline & Q Position Defense & & BRANDING STRATEGIES & प Specialty Store & $\square$ Print - Newsletter & \\
\hline & $\square$ Flank Defense & & Brand Development Objective & a Catalog Showroom & $\square$ Print - Brochure & \\
\hline & a Premptive Defense & & $\square$ Line Extension & $\square$ Own Outlet & $\square$ Outdoor - Billboards & \\
\hline CUSTOMER SEGMENT & D Counteroffensive Defense & & D Brand Extension & a Something New & Outdoor - POP & \\
\hline Geographic & $\square$ Mobile Defense & & 0 Multi-brand & PRICING STRATEGIES & $\square$ Direct mail & \\
\hline Q Region & D Contraction Defense & & D New Brand & Pricing Objective & Telephone & \\
\hline$\nabla$ City & Challenger Strategies & & Brand Sponsorship Objective & 0 Maximize Market share & Intemet & \\
\hline$\square$ Density & $\checkmark$ Frontal attack & & Q Private Label & D Maximize Profit & Digital Media Tools & \\
\hline$\square$ Climate & Q Flank attack & & Q Licensed Brand & I Survival & D Facebook & \\
\hline Demographic & - Encirclement attack & & $\square$ Co-Brand & Competitor's Prices & $\square$ Instagram & \\
\hline$\square$ Age & $\square$ Bypass attack & & Brand Portfolio Role & Competitor $\# 1$ & D Twitter & \\
\hline$\square$ Gender & Q Guerilla warfare & & $\begin{array}{l}\text { - Flankers } \\
\end{array}$ & Competitor $\# 2$ & D YouTube & \\
\hline$\square$ Family size & Follower Strategies & & 0 Cash cows & Competitor \#3 & $\square$ Snapchat & \\
\hline$\square$ Family Life cycle & $\square$ Counteffeiter & & \begin{tabular}{|l|} 
Low-end entry level \\
\end{tabular} & Competitor $\# 4$ & D Google Map & \\
\hline Q Race & $\square$ Cloner & & $\square$ High-end prestige & Retail Price & Sales Promotion T & \\
\hline D Occupation & Imitator & & Brand Equity Tools & Retail Price per unit & $\square$ Samples Free trials & \\
\hline$\square$ Income & a Adapter & & Q Brand Reinforcement & Wholesale Price per unit & $\square$ Coupons & \\
\hline Q Education & \begin{tabular}{|l|} 
COMPETITIVE TOOLS \\
\end{tabular} & & Q Brand Revitalization & Cost per Unit & D Cash refund offers & \\
\hline \begin{tabular}{|l|} 
Psychographic \\
\end{tabular} & $\square$ Price discounts & & $\square$ Brand Activations & Promotional Pricing & Q Price offs & \\
\hline$\square$ Life Style & Q Lower-priced goods & & $\square$ Brand Extensions & $\square$ Loss-leader pricing & $\square$ Premiums & \\
\hline \begin{tabular}{|l|} 
Personality \\
\end{tabular} & Q Value-priced goods & & Branding Elements & - Special-event pricing & $\square$ Prizes & \\
\hline Behavioral & $\square$ Prestige goods & & Brand Naming & 0 Cash rebates & Q Loyalty rewards & \\
\hline$\square$ Occasions & $\square$ Product proliferation & & I Individual names & Q Low-interest financing & $\square$ Tie-in promotions & \\
\hline$\square$ Benefits & $\checkmark$ Product innovation & & Q Blanket family names & $\square$ Longer payment tems & PR Tools & \\
\hline IUses & I Improved services & & ¿ Separate family names & - Warranties \& service & $\square$ Press Release & \\
\hline$\square$ Attitudes & a Distribution innovation & & 0 Corporate individual name combo & D Psychological discounting & I CEO Speeches & \\
\hline CUSTOMER PROFILE & D Manufacturing-cost reduction & & QuRLs & Discounts & Q Public service activities & \\
\hline$\square$ Age & Intensive advertising promotion & & $\square$ Logo & Q Cash discount & Sales Force & \\
\hline$\square$ Gender & Increase users & & DCharacter & Q Quantity discount & Number of Sales Stafff & \\
\hline$\square$ Income & D Increase usage & & D Slogan & $\square$ Functional discount & Commission \& Benefits & \\
\hline \begin{tabular}{|l|}
$\square$ Social Status \\
\end{tabular} & Increase usage & & \begin{tabular}{|l|} 
\\
\end{tabular} & $\square$ Seasonal discount & Event Sponsorship & \\
\hline$\square$ Preference & D Something New & & $\square$ Something New & 0 Trade Allowance & Number of Events & \\
\hline Total Sale & Expected (Number of units) & & & Promotion Budget & & \\
\hline Total Sales Proceeds & umber of Units X Unit Price) & & Tot & Discounts offered & & \\
\hline Total Cost (Nun & per of units sold $X$ Unit Cost) & & & tal Marketing Cost & & \\
\hline
\end{tabular}

Figure 1. Marketing Model Canvas as an Assessment Tool for Marketing Curriculum

\subsection{Results}

Marketing Model Canvas has ten sections (Figure 1):

1. Marketing Objective outlines the purpose of the marketing plan and provides a clear direction for marketing team to follow, review and support.

2. including launching a new line extension, brand extension, increasing market penetration,

3. Customers including customer needs, wants, segment and profile.

4. Competitors including major competitors, strategies for competition including leaders, challengers and followers' strategies and competitive tools.

5. Value Proposition including unique selling proposition, positioning objective and perceptual maps.

6. Product and Branding including product line objectives, product mix description, brand development objectives, brand sponsorship objectives, branding elements brand equity measures and brand portfolio role.
7. Place and Distribution including distribution objectives and distribution network.

8. Pricing including pricing objectives, competitor's prices, retail price, promotional pricing, and discounts.

9. Promotion and Advertising includes advertising objectives, advertising media selection, digital media tools, sales promotion tools, PR tools, Sales Force utilization and event sponsorship.

10. Financial Implications where the cost stream (what is incurred to operate the marketing model) and the revenue stream (the cash gained from each customer segments) should be considered.

\subsection{Rubrics Development}

In order to enhance the effective utilization of the Marketing Model Canvas, rubrics were developed (Table 5) and tested for student's assessments of their Marketing Model presentations. Three important points need to be considered while using these rubrics. Firstly, these rubrics are effective for all three conditions for a Marketing 
Model Canvas may be considered (a) marketing plan for a live-client, (b) marketing plan using a written case setting, or (c) marketing plan for a real product using publicly available information or data. Secondly these rubrics are developed for general purpose marketing plans, for services marketing plans small adjustments needs to be made before announcements of rubrics. Financial implications sections may be omitted for undergraduate students.

\subsection{Benefits}

From the above exercise few observations can easily be made; (a) Marketing Model Canvas provides a new way for marketing academics to include one-page, all-inclusive assessment tool as part of any marketing subject; (b) Students found it easier to complete their market plan assignments using this template but with greater depth and with greater ease.

Table 5. Marketing Model Canvas - Rubrics for Assessments

\begin{tabular}{|c|c|c|c|}
\hline Criteria & $\begin{array}{c}\text { Unsatisfactory } \\
\text { (0-1 Marks) }\end{array}$ & $\begin{array}{l}\text { Satisfactory } \\
\text { (2-3 Marks) }\end{array}$ & $\begin{array}{c}\text { Excellent } \\
(4-5 \text { Marks })\end{array}$ \\
\hline $\begin{array}{l}\text { Marketing } \\
\text { Objective }\end{array}$ & $\begin{array}{l}\text { Marketing objective is missing or } \\
\text { not quantifiable. }\end{array}$ & $\begin{array}{l}\text { Marketing objective is unclear and/or } \\
\text { not appropriately quantified. }\end{array}$ & $\begin{array}{l}\text { Marketing objective is clearly stated and } \\
\text { appropriately quantified. }\end{array}$ \\
\hline Customer & $\begin{array}{l}\text { Little or no detail provided on the } \\
\text { customer needs and wants, its } \\
\text { market segments and customer } \\
\text { profiles. }\end{array}$ & $\begin{array}{l}\text { Provides an incomplete description of } \\
\text { customer needs and wants; } \\
\text { inappropriate customer segments and/or } \\
\text { incomplete customer profile. }\end{array}$ & $\begin{array}{l}\text { An outstanding assessment that clearly } \\
\text { identifies the target market, its } \\
\text { characteristics and needs, and utilizes } \\
\text { secondary sources to support this } \\
\text { assessment. }\end{array}$ \\
\hline Competitor & $\begin{array}{c}\text { Major competitors are not } \\
\text { identified; competitive strategies } \\
\text { are not selected; competitive tools } \\
\text { are checked. }\end{array}$ & $\begin{array}{l}\text { Major competitors are identified but not } \\
\text { correct or the irrelevant market key } \\
\text { players are identified; competitive } \\
\text { strategies are selected but not relevant; } \\
\text { inappropriate competitive tools are } \\
\text { selected. }\end{array}$ & $\begin{array}{l}\text { Excellent competitor analysis: major } \\
\text { competitors are correctly identified and } \\
\text { relevant market key players are } \\
\text { identified; competitive strategies are } \\
\text { correctly selected; appropriate } \\
\text { competitive tools are selected. Make } \\
\text { sure only type of competitive strategy } \\
\text { has been selected from leader, } \\
\text { challenger, or follower strategies. }\end{array}$ \\
\hline $\begin{array}{l}\text { Value } \\
\text { Proposition }\end{array}$ & $\begin{array}{l}\text { Little to no information is provided } \\
\text { on the positioning objectives, USP } \\
\text { of the product /service, its features } \\
\text { and benefits, its perceptual maps. }\end{array}$ & $\begin{array}{c}\text { Positioning objectives are mentioned } \\
\text { but not correct, Inappropriate USP of } \\
\text { the product /service is mentioned, } \\
\text { perceptual maps are based on incorrect } \\
\text { bases. }\end{array}$ & $\begin{array}{l}\text { Appropriate positioning objectives are } \\
\text { mentioned, USP of the product /service } \\
\text { is relevant, excellent perceptual maps } \\
\text { are drawn using appropriate bases. }\end{array}$ \\
\hline $\begin{array}{l}\text { Product } \\
\text { Strategies }\end{array}$ & $\begin{array}{l}\text { No details are given about product } \\
\text { line objectives and/or product mix. }\end{array}$ & $\begin{array}{l}\text { Incorrect details are given about product } \\
\text { line objectives and/or product mix. }\end{array}$ & $\begin{array}{c}\text { Appropriate product line objectives are } \\
\text { mentioned and correct details of product } \\
\text { mix are given. }\end{array}$ \\
\hline $\begin{array}{l}\text { Branding } \\
\text { Strategies }\end{array}$ & $\begin{array}{l}\text { Branding objectives are not } \\
\text { mentioned; branding tools are not } \\
\text { identified, brand portfolio role not } \\
\text { mentioned, branding elements are } \\
\text { not selected. }\end{array}$ & $\begin{array}{l}\text { Branding objectives are mentioned; } \\
\text { branding tools are identified, brand } \\
\text { portfolio role is mentioned, branding } \\
\text { elements are selected but some } \\
\text { missing's are there. }\end{array}$ & $\begin{array}{l}\text { Appropriate branding objectives are } \\
\text { mentioned; correct branding tools are } \\
\text { identified, appropriate brand portfolio } \\
\text { role is mentioned, reasonable branding } \\
\text { elements are selected. }\end{array}$ \\
\hline $\begin{array}{c}\text { Place } \\
\text { Strategies }\end{array}$ & $\begin{array}{l}\text { Distribution objectives are not } \\
\text { mentioned; distribution network is } \\
\text { not selected. }\end{array}$ & $\begin{array}{l}\text { Distribution objectives are not in line } \\
\text { with PLC stage; inappropriate } \\
\text { distribution channel is selected. }\end{array}$ & $\begin{array}{l}\text { Appropriate distribution objectives are } \\
\text { mentioned and adequate distribution } \\
\text { channel is selected. }\end{array}$ \\
\hline $\begin{array}{l}\text { Pricing } \\
\text { Strategies }\end{array}$ & $\begin{array}{c}\text { Pricing objectives are not } \\
\text { mentioned, competitor's prices not } \\
\text { mentioned, retail price details not } \\
\text { mentioned, promotional priming } \\
\text { tools and discounting tools are not } \\
\text { selected. }\end{array}$ & $\begin{array}{l}\text { Pricing objectives are not in line with } \\
\text { PLC stage, competitor's prices vary, } \\
\text { incomplete retail price details are } \\
\text { mentioned, inappropriate promotional } \\
\text { priming tools and discounting tools are } \\
\text { selected. }\end{array}$ & $\begin{array}{l}\text { Pricing objectives are in line with PLC } \\
\text { stage, competitor's prices and retail price } \\
\text { details are in line with each other, } \\
\text { appropriate promotional priming tools } \\
\text { and discounting tools are selected and } \\
\text { amount of discount mentioned. }\end{array}$ \\
\hline $\begin{array}{l}\text { Promotion } \\
\text { Strategies }\end{array}$ & $\begin{array}{c}\text { Advertising objectives are not } \\
\text { mentioned, advertising media is } \\
\text { missing, digital media tools are } \\
\text { missing, sales promotional tools } \\
\text { and PR tools are not selected, sales } \\
\text { force and event sponsorship details } \\
\text { are ignored. }\end{array}$ & $\begin{array}{c}\text { Advertising objectives are not in line } \\
\text { with the PLC stage, wrong advertising } \\
\text { media is selected, inappropriate digital } \\
\text { media tools are selected, inappropriate } \\
\text { sales promotional tools and PR tools are } \\
\text { selected, incomplete sales force and } \\
\text { event sponsorship details are } \\
\text { mentioned. }\end{array}$ & $\begin{array}{l}\text { Advertising objectives are in line with } \\
\text { the PLC stage, adequate advertising } \\
\text { media is selected, appropriate digital } \\
\text { media tools are selected, appropriate } \\
\text { sales promotional tools and PR tools are } \\
\text { selected, sales force and event } \\
\text { sponsorship details are mentioned. }\end{array}$ \\
\hline $\begin{array}{c}\text { Financial } \\
\text { Implications }\end{array}$ & $\begin{array}{l}\text { No calculation given for cost } \\
\text { stream and/or revenue stream. }\end{array}$ & $\begin{array}{l}\text { Incorrect calculations were presented } \\
\text { for cost stream and/or revenue stream. }\end{array}$ & $\begin{array}{l}\text { Reasonable calculations were presented } \\
\text { for cost stream and/or revenue stream. }\end{array}$ \\
\hline
\end{tabular}




\section{Conclusions}

Despite its limitations, the findings of this study provide a new way for marketing academics to include this one-page, all-inclusive Marketing Model Canvas as part of their marketing subject. Students will also find it easier to complete their market plan assignments using this template but with greater depth. The study produces various implications for social scientists especially those who would like to convert instruments in their research or those who would like to develop new instruments to measure something with an old reliable construct for a new discipline.

\section{REFERENCES}

[1] Rohm, A. J., Stefl, M., \& Saint Clair, J. (2019). Time for a marketing curriculum overhaul: Developing a digital-first approach. Journal of Marketing Education, 41(1), 47-59.

[2] Wharton Business School https://marketing.wharton.upenn. edu/brief-history-wharton-marketing-department/\#: :text=T he $\% 20$ first $\% 20$ marketing $\% 20$ course $\% 2 \mathrm{C} \% 20 \% \mathrm{E} 2 \% 80 \% 9$ CMarketing,advertising\%20and\%20salesmanship \%20in\%2 01909.\&text=Breyer's\%20work\%20on $\% 20 \% \mathrm{E} 2 \% 80 \% 9 \mathrm{Cch}$ annels $\%$ E2\%80\%9D\%20became,of $\% 20$ marketing\%20studi es $\% 20$ at $\% 20$ Wharton.

[3] Ferrell, O. C., Hair Jr, J. F., Marshall, G. W., \& Tamilia, R. D. (2015). Understanding the history of marketing education to improve classroom instruction. Marketing Education Review, 25(2), 159-175.)

[4] Harvard Business School https://online.hbs.edu/blog/post/th e-history-of-the-case-study-at-harvard-business-school

[5] Association to Advance Collegiate Schools of Business AACSB (2021) https://www.aacsb.edu/insights/2019/may/c ourse-correction-recalibrating-experiential-learning-in-themba

[6] Harvard Business School https://online.hbs.edu/blog/post/th e-history-of-the-case-study-at-harvard-business-school

[7] Association to Advance Collegiate Schools of Business AACSB (2021) https://www.aacsb.edu/insights/2019/may/c ourse-correction-recalibrating-experiential-learning-in-themba

[8] Westwood, J. (2002). The marketing plan: a step-by-step guide. Kogan Page Publishers.

[9] Association to Advance Collegiate Schools of Business AACSB (2021) https://www.aacsb.edu/insights/2019/may/c ourse-correction-recalibrating-experiential-learning-in-themba

[10] Lincoln, D. J., \& Frontczak, N. T. (2008). A practical and effective marketing plan assignment for principles of marketing students. Journal for Advancement of Marketing Education, 12, 52-62.

[11] Marketing Plan - Harvard Business School https://canvas.harvard.edu/courses/2223/assignments/syllab us

[12] Marketing Plan - DeGroote School of Business, McMaster University, Canada https://ug.degroote.mcmaster.ca/files/20 19/08/3MC3-C05-2019-Fall-Course-Outline-P-McClellandfinal.pdf

[13] Marketing Plan - Rutgers School of Business - Camden Rutgers University, USA https://business.camden.rutgers.ed u/wp-content/uploads/2020/06/52-630-403-40-Strategic-Ma rket-Planning-FINK.pdf

[14] Marketing Plan - University of South Australia, Australia https://my.unisa.edu.au/public/courseoutline/ViewOutline.as px?id=29612

[15] Marketing Plan - IESE Business School, Spain https:/globalexecutivemba.iese.edu/wp-content/uploads/M4 -Marketing-Planning-and-Implementation.pdf

[16] Marketing Plan - Victoria University of Wellington, New Zealand https://www.wgtn.ac.nz/business/current-students/study-res ources/course-outlines/2011/trimester-2/mark/2011_2_mark 302.pdf

[17] Marketing Plan - Western University, Canada https://dan.uwo.ca/documents/course_information/2014-201 5/MOS9320A001Cleveland1.pdf

[18] Marketing Plan - Victoria University of Wellington, New Zealand

https://www.wgtn.ac.nz/courses/emba/506/2020/offering?cr $\mathrm{n}=32163$

[19] Marketing Plan - Sheridan College, Canada http://ulysses.sheridanc.on.ca/coutline/coutlineview.jsp?prin $\mathrm{t}=$ true \& courseCode $=27583 \&$ subjectCode $=$ MKTG\&version $=9.0 \&$ appver $=\mathrm{ps}$

[20] Marketing Plan - University of Canterbury, Canada https://www.canterbury.ac.nz/courseinfo/GetCourseDetails. aspx? course $=$ MKTG311 \&occurrence $=17$ S1 $($ C $)$ \&year $=2017$

[21] Marketing Plan - Georgian College, Canada https://www.ge orgiancollege.ca/course-outlines/MKTG/2001/Fall/2014

[22] Osterwalder, A. (2004). The business model ontology a proposition in a design science approach (Doctoral dissertation, Université de Lausanne, Faculté des hautes études commerciales).

[23] Osterwalder, A., \& Pigneur, Y. (2010). Business model generation: a handbook for visionaries, game changers, and challengers. John Wiley \& Sons.

[24] Osterwalder, A., Pigneur, Y., \& Tucci, C. L. (2005). Clarifying business models: Origins, present, and future of the concept. Communications of the association for Information Systems, 16(1), 1.

[25] Kotler, P., \& Keller, K. L. (2018). Marketing Management, Global Edition (15 $5^{\text {th }}$ ed). Harlow.

[26] Whitler, K. (2018). Developing a Superior Strategic Marketing Plan. Harvard Business School Publishing. https://hbsp.harvard.edu/product/UV7559-PDF-ENG0

[27] Spekman, R. E. (2013). Marketing Plan Development. 
Harvard Business School Publishing. https://hbsp.harvard.e du/product/UV6731-PDF-ENG
[28] Calkins, T., (2006) Writing Great Marketing Plans. Harvard Business School Publishing. https://hbsp.harvard.edu/produc t/908A15-PDF-ENG 